\begin{tabular}{|c|c|}
\hline Title & Theoretical study on electronic states of potassium-oxygen-graphite ternary intercal ation compounds \\
\hline Author(s) & Higai, Shin'ichi; Mizuno, Seiji; Nakao, Kenji \\
\hline Citation & $\begin{array}{l}\text { Physical Review B, } 57(12), 7369-7376 \\
\text { https://doi.org/10.1103/PhysRevB.57.7369 }\end{array}$ \\
\hline Issue Date & $1998-03$ \\
\hline DOC URL & http:/hdl.handle.net/2115/50998 \\
\hline Rights & (c) 1998 The A merican Physical Society \\
\hline Type & article \\
\hline File Information & Phys. Rev. B 57, 7369.pdf \\
\hline
\end{tabular}

Instructions for use 


\title{
Theoretical study on electronic states of potassium-oxygen-graphite ternary intercalation compounds
}

\author{
Shin'ichi Higai* \\ Institute of Materials Science, University of Tsukuba, Tsukuba 305-8573, Japan \\ Seiji Mizuno \\ Department of Applied Physics, Hokkaido University, Sapporo 060-8628, Japan \\ Kenji Nakao \\ Institute of Materials Science, University of Tsukuba, Tsukuba 305-8573, Japan
}

(Received 25 August 1997)

\begin{abstract}
The electronic states of stage-1 and -2 potassium-oxygen-graphite ternary intercalation compounds are studied from first principles by constructing several simple structural models. For all models, the graphite $\pi^{*}$ band and the oxygen $2 p$ band form the electron and hole Fermi surfaces, respectively. Oxygen $2 p$ bands with small dispersion contribute to the large density of states near the Fermi level. The whole $4 s$ charge of potassium is transferred to oxygen and carbon, and the present compounds are classified into the donor type. Furthermore, no magnetic moment appears on oxygen irrespective of its arrangement, for which the reason is discussed. [S0163-1829(98)03912-5]
\end{abstract}

\section{INTRODUCTION}

A new type of ternary graphite intercalation compounds (GIC's), alkali-metal GIC's containing oxygen, was reported recently. ${ }^{1,2}$ In these reports, an introduction of a small amount of oxygen in alkali-metal GIC's gives rise to a high content of alkali metal by forming a multilayered structure within galleries between layers of host graphite. As for an alkali metal and oxygen, they form three types of ionic crystals, which are alkali-metal monoxides $\left(A_{2} \mathrm{O}\right)$, peroxides $\left(A_{2} \mathrm{O}_{2}\right)$, and hyperoxides $\left(A \mathrm{O}_{2}\right)(A=\mathrm{Na}, \mathrm{K}, \mathrm{Rb}, \mathrm{Cs})$. While the former two show diamagnetism, alkali-metal hyperoxides exhibit antiferromagnetism due to the coupling of inherent spins of the diatomic molecular hyperoxide ions $\mathrm{O}_{2}{ }^{-}$. Therefore, GIC's containing $\mathrm{O}_{2}{ }^{-}$were expected to exhibit the magnetism associated with an interaction between conduction $\pi$ electrons of graphite and localized magnetic moments of $\mathrm{O}_{2}{ }^{-}$. With this expectation, Mordkovich et al. ${ }^{3}$ synthesized and isolated stage- 1 and -2 potassium-oxygen GIC's (KO-GIC's) $\left(\mathrm{C}_{4 n} \mathrm{KO}_{2}\right.$, where $n$ is the stage number) with the use of potassium hyperoxides $\left(\mathrm{KO}_{2}\right)$. They studied the crystal structure of KO-GIC's based on an X-raydiffraction intensity analysis and the transmission electron microscopy electron diffraction, and supposed that two intercalating species, potassium and oxygen, form separate quadruple $\mathrm{K}-\mathrm{O}-\mathrm{O}-\mathrm{K}$ layers between graphite layers. As concerns the electronic states, Yamashita et $a .^{4}{ }^{4}$ carried out magneticsusceptibility measurements for KO-GIC's. Contrary to the above expectation, their results showed that oxygen in $\mathrm{KO}-$ GIC's is in a nonmagnetic state. They also estimated the density of states (DOS) at the Fermi level $\left(E_{F}\right)$ from the Pauli paramagnetic components, and showed that these are much larger than those expected from only the graphite $\pi$ band. This fact suggests the participation of the delocalized electronic states of intercalates at $E_{F}$. In addition, the Hall- effect measurements for KO-GIC's showed that the signs of their Hall coefficients are positive. ${ }^{5}$ This suggests the existence of hole carriers originating from the intercalate bands.

To understand the electronic properties of KO-GIC's systematically, theoretical studies are required. In the present work, we calculate the electronic band structures of KOGIC's from first principles in order to obtain a basis for understanding their electronic properties. In particular, we focus our attention on the following three points. The first point is the question of whether KO-GIC's are classified into donor or acceptor types, because the donor (potassium) and acceptor (oxygen) of electrons coexist in these compounds. The second point is related to the origin of the electronic states at $E_{F}$. The last point is to obtain the net spin polarization on oxygen atoms in the compounds, in connection with the experimental fact of the disappearance of the magnetic moment in KO-GIC's.

The outline of this paper is as follows. In Sec. II, we construct several structural models based on the experimental results. The method of calculation is briefly described in Sec. III. The resulting band structures are shown in Sec. IV. From them, we extract the DOS, cross section of the Fermi surface (FS), carrier density, and amount of the charge transfer. In Sec. V, we discuss several experimental results in terms of our results. Here the reason for the disappearance of the magnetic moment is also explained. A summary is given in Sec. VI.

\section{CRYSTAL STRUCTURE}

Experimental results concerning the crystal structures of KO-GIC's are summarized as follows. ${ }^{3,4,6}$ The chemical formulas for the stage- 1 and -2 compounds are $\mathrm{C}_{4} \mathrm{KO}_{2}$ and $\mathrm{C}_{8} \mathrm{KO}_{2}$, respectively. The $c$-axis repeat distances $I_{c}$ differ with synthesis methods. For samples synthesized with the vapor phase method, $I_{c}$ 's for the stage- 1 and -2 compounds 
are 8.21 and $11.62 \AA$, respectively. However, for samples synthesized with the liquid phase method, $I_{c}$ 's are 8.44 and $11.72 \AA$ for the stage- 1 and -2 compounds, respectively. The intercalated potassium and oxygen form separate quadruple layers. The stacking order along the $c$ axis is (C)-C-K-O-O$\mathrm{K}-\mathrm{C}-(\mathrm{C})$, where the parentheses are for stage 2 . The structure of a carbon layer is a hexagonal net as in graphite, but distances between the nearest-neighbor carbon atoms $\left(d_{\mathrm{C}-\mathrm{C}}\right)$ are expanded compared with that of pristine graphite. $d_{\mathrm{C}-\mathrm{C}}$ 's for the stage- 1 and -2 compounds, and pristine graphite are $1.431,1.4260$, and $1.4216 \AA$, respectively. In a potassium layer, potassium atoms form the close-packed $(2 \times 2)$ triangular lattice structure. The distance between two potassium layers is $2.73 \pm 0.12 \AA$. On the other hand, the arrangement of oxygen atoms is uncertain, although the existence of two oxygen layers is detected.

In the present study, we construct three simple structural models with the smallest unit cell as follows. First of all, there is a possibility that oxygen atoms are intercalated as $\mathrm{O}_{2}$ molecules, because $\mathrm{KO}_{2}$ used for the synthesis of KO-GIC's is composed of $\mathrm{K}^{+}$cations and molecular $\mathrm{O}_{2}{ }^{-}$anions. In this case, two further arrangements are possible, i.e., bond axes of the $\mathrm{O}_{2}$ molecules are parallel (model A) or perpendicular (model B) to the $c$ axis. Models A and B are shown in Figs. 1(a) and 1(b), respectively. The $z$ axis is chosen to be parallel to the $c$ axis. In model $\mathrm{A}$, the distance between adjacent oxygen layers is chosen to be $1.207 \AA$, which corresponds to the bond length of an isolated $\mathrm{O}_{2}$ molecule. In model $\mathrm{B}$, this distance is chosen to be $1.94 \AA$. The bond lengths of the $\mathrm{O}_{2}$ molecules are $1.207 \AA$, as in model $\mathrm{A}$, and their bond axes are also perpendicular to the direction joining the two nearest-neighbor potassium atoms. This arrangement is determined so that the distance between neighboring potassium and oxygen atoms is close to that in the $\mathrm{KO}_{2}$ crystal, on the ground that two negatively charged oxygen layers repel each other, and they are attracted by positively charged potassium layers.

Furthermore, there is another possibility that oxygen atoms are intercalated as nearly isolated atoms (model C). As mentioned in Sec. I, the results of the magnetic-susceptibility measurements suggest a nonmagnetic state of oxygen. We consider this model to ascertain whether the reason for the disappearance of the magnetic moment on oxygen is the dissociation of the $\mathrm{O}_{2}$ molecule in these compounds. Model C is presented in Fig. 1(c). The top view of this model is the same as that of model A, shown in Fig. 1(a), but the distance between adjacent oxygen layers is extended. This distance is tentatively settled to $1.94 \AA$ as in model B.

In Fig. $1, d_{i}$ represents the distance between two graphite layers which sandwich intercalate layers. For the stage-1 compound, $d_{i}$ is just equal to $I_{c}$, while, for the stage- 2 compound, $d_{i}$ has not been experimentally obtained. We assume $d_{i}$ for the stage- 2 compound to be equal to that for the stage-1 compound, because the difference between $I_{c}$ 's for the stage- 1 and -2 compounds is close to the interlayer distance of the pristine graphite $(3.35 \AA)$.

Carbon atoms in GIC's are distinguished by the environment nonequivalency. These are denoted by numbers 1 and 2 (and 3 for stage 2) in Fig. 1. We treat these carbon atoms distinctively, because the charge imbalance among the nonequivalent carbon atoms in the graphite layer is pointed out to be important on the electronic structures of GIC's in previous papers. ${ }^{7,8}$

Figure 2 shows the Brillouin zone (BZ) for our structural models, where the high-symmetry points and lines are labeled. In this BZ, all $K$ points are equivalent, but the points denoted $M_{1}$ and $M_{2}$ are distinguished.

\section{METHOD OF CALCULATION}

In the present study, we adopted the linear combination of atomic orbitals (LCAO) method within the local-spin-density approximation (LSDA) ${ }^{9-11}$ The LCAO method we used has some advantages. In this method, the partial DOS can be clearly defined. Therefore, it is useful to understand the characteristics of energy bands in investigating the electronic structures of complicated materials such as GIC's. Moreover, the integration of the partial DOS directly gives the populations of the atomic orbitals. Thereby, we can estimate the amount of the charge transfer for constituent atoms. The charge transfer plays an essential role in determining the electronic structures of GIC's. Furthermore, the calculational compactness of the LCAO method enables us to manage large systems. For the basis functions, we used the selfconsistent all-electron numerical basis set. ${ }^{72}$ In the selfconsistent calculation, the populations of the atomic orbitals are utilized for the parameters of the iteration. Based on them, the basis functions are modified in every iterative step to be consistent with the corresponding charge density. In order to sample the BZ, we took $576 k$ points in the irreducible BZ. For the exchange-correlation potential, the PerdewZunger parametrization ${ }^{11}$ is used.

\section{BAND STRUCTURE, FERMI SURFACE, AND CHARGE TRANSFER}

We have carried out a band-structure calculation for the stage- 1 compound $\mathrm{C}_{4} \mathrm{KO}_{2}$ and stage- $2 \mathrm{C}_{8} \mathrm{KO}_{2}$ with the structural models $\mathrm{A}, \mathrm{B}$, and $\mathrm{C}$. The electronic structures were determined for two different $c$-axis repeat distances $I_{c}$, given in Sec. II. However, we saw little difference between the results calculated for these two $I_{c}$ 's. Moreover, the stage number dependences seen in the results were similar for models $\mathrm{A}, \mathrm{B}$, and $\mathrm{C}$. In this section, we show the results for $\mathrm{C}_{4} \mathrm{KO}_{2}$ with the structural models $\mathrm{A}, \mathrm{B}$, and $\mathrm{C}$, and $\mathrm{C}_{8} \mathrm{KO}_{2}$ with the model A, which are calculated with the use of $I_{c}$ for the sample synthesized with the vapor phase method.

\section{A. Stage-1 compound with structural model A}

First, in Fig. 3(a), we present the band structure and corresponding partial DOS calculated for the stage- $1 \mathrm{C}_{4} \mathrm{KO}_{2}$ with structural model $\mathrm{A}$, in which oxygen atoms are intercalated as $\mathrm{O}_{2}$ molecules whose bond axes are parallel to the $c$ axis.

As the first step to determine the electronic structure, we carried out a spin-independent band-structure calculation. Then we also made a spin-dependent calculation based on the LSDA, in order to examine the amount of the spin polarization on the $\mathrm{O}_{2}$ molecules in the compound. Our results show that the spin-dependent band structure is nearly the same as the spin-independent one, and the $\mathrm{O}_{2}$ molecules have no magnetic moment in the compound within the accu- 
(a)

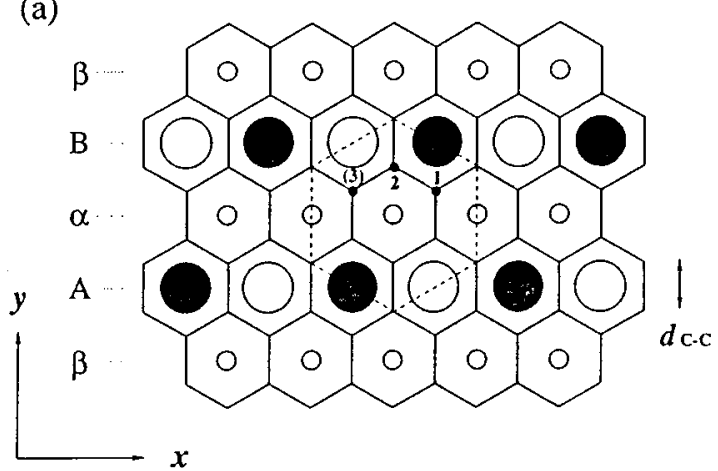

(b)
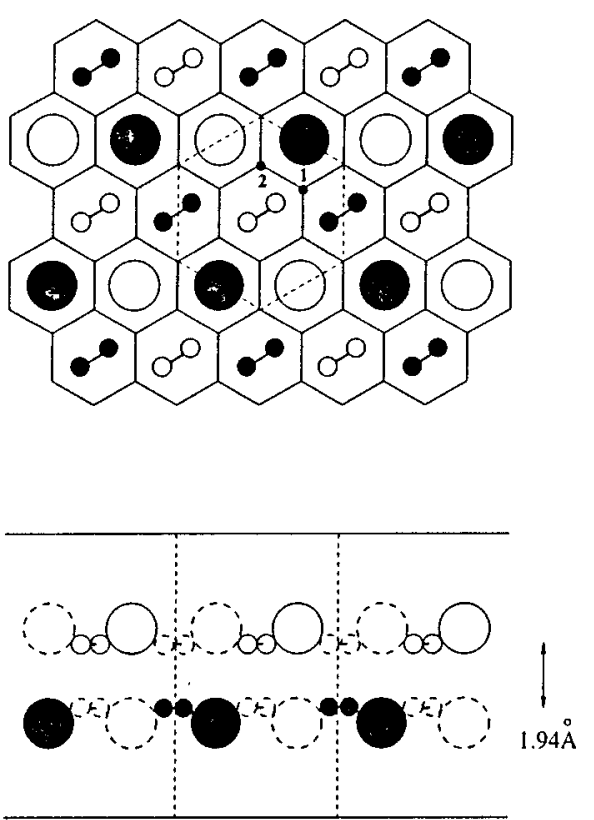

(c)
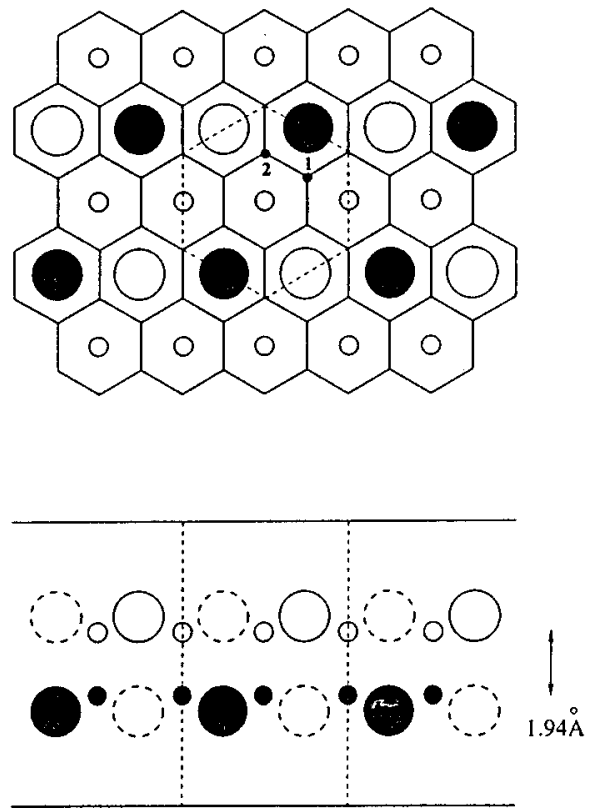

FIG. 1. The top and side views of the structural model: (a) model A, (b) model B, and (c) model C. Open and closed large circles represent potassium atoms, and open and closed small circles represent oxygen atoms. A unit cell is shown by dashed lines. The labels $A$ and $B$ indicate the rows of potassium atoms along the $x$-axis direction, and $\alpha$ and $\beta$ indicate those of oxygen atoms. In the side view, the rows of $B$ and $\beta$ are shown by dashed circles.

racy of the present numerical calculation. The reason for the disappearance of the magnetic moment is discussed in Sec. V.

The overall band structure shown in Fig. 3(a) can be understood as the superposition of potassium and oxygen bands on graphite bands. That is, the effect of hybridization of the band with a different character is rather small. As a result, each of the dispersion curves shown in Fig. 3(a) can be identified with the bonding $\pi$ and antibonding $\pi^{*}$ bands of graphite, the $2 p$ bands of oxygen, and the $4 s$ bands of po- tassium. Though the $\pi$ and $\pi^{*}$ bands have nearly the same structures as those of two-dimensional graphite folded into the BZ for this model, the charge imbalance in the graphite layer generated by the crystal field causes the splittings of the $\pi$ and $\pi^{*}$ bands. These are clearly seen along the $\Gamma-M_{1}$ direction around -10 and $-5 \mathrm{eV}$. As for the potassium bands, two $4 s$ bands lie in the energy region -0.4 to $3.0 \mathrm{eV}$, much higher than $E_{F}$.

The most noticeable feature of this band structure is the existence of four $2 p$ bands of oxygen with small dispersion 

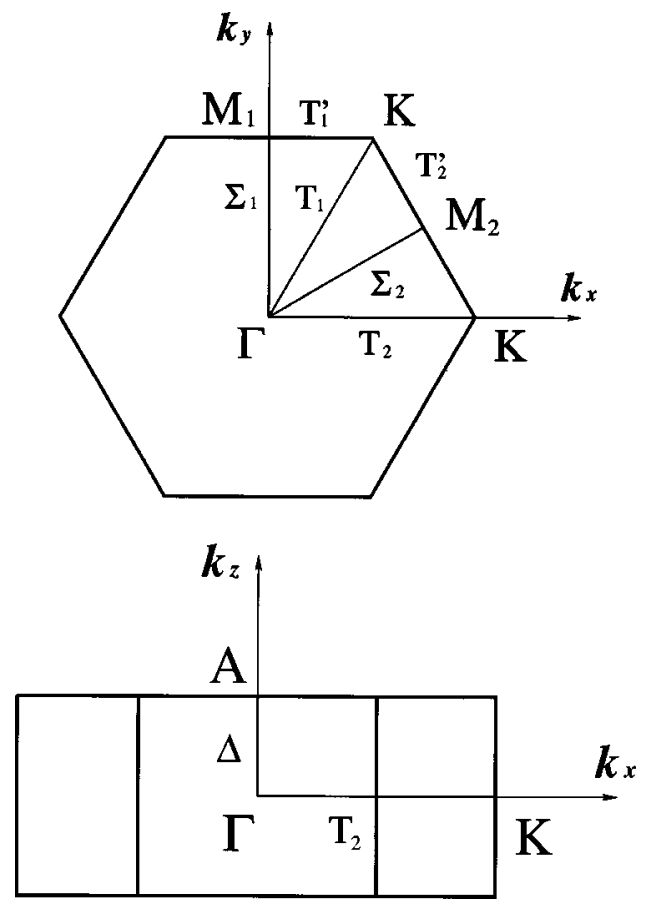

FIG. 2. The Brillouin zone for $\mathrm{C}_{4} \mathrm{KO}_{2}$ and $\mathrm{C}_{8} \mathrm{KO}_{2}$.

near $E_{F}$. In the oxygen $2 p$ bands, the character of the $\mathrm{O}_{2}$ molecular orbitals (MO's) is reflected. These four $2 p$ bands near $E_{F}$ are identified as the $2 p_{x} \pi^{*}, 2 p_{y} \pi^{*}, 2 p_{y} \pi^{*}$, and $2 p_{x} \pi^{*}$ bands from the higher-energy side at the $\Gamma$ point, respectively. Furthermore, there exist two $2 p_{z} \sigma^{*}$ bands around $7 \mathrm{eV}$, beyond this figure, and four $2 p \pi$ and two $2 p \sigma$ bands in -14.1 to $-10.5 \mathrm{eV}$. Concerning the $2 p_{x} \pi^{*}$ and $2 p_{y} \pi^{*}$ bands, they split due to the interaction between the nearest $\mathrm{O}_{2}$ molecules. This splitting and the bandwidths of
TABLE I. The total DOS at the Fermi level.

\begin{tabular}{|c|c|c|c|c|}
\hline$\langle$ theory $\rangle$ & model A & $\begin{array}{c}\mathrm{C}_{4} \mathrm{KO}_{2} \\
\text { stage } 1 \\
\text { model } \mathrm{B}\end{array}$ & model C & $\begin{array}{c}\mathrm{C}_{8} \mathrm{KO}_{2} \\
\text { stage } 2 \\
\text { model A }\end{array}$ \\
\hline $\begin{array}{l}\text { DOS } \\
\left(\times 10^{22} \text { states } / \mathrm{eV} / \mathrm{cm}^{3}\right)\end{array}$ & 3.09 & 2.47 & 1.54 & 2.47 \\
\hline$\langle$ experiment $\rangle$ & & & & \\
\hline $\begin{array}{l}\text { DOS } \\
\left(\times 10^{22} \text { states } / \mathrm{eV} / \mathrm{cm}^{3}\right)\end{array}$ & & 2.1 & & 1.3 \\
\hline
\end{tabular}

$2 p_{x} \pi^{*}$ bands are larger than those of $2 p_{y} \pi^{*}$ bands, reflecting the one-dimensional arrangement of oxygen atoms in model A. In addition to the graphite $\pi^{*}$ bands, these $2 p \pi^{*}$ bands of oxygen contribute to the large DOS around $E_{F}$, where the hybridization of potassium $4 s$ bands is hardly seen [see the partial DOS shown in Fig. 3(a)]. The value of the total DOS at $E_{F}$ is shown in Table I to compare with the experimental one.

In the intralayer directions $\left(K-\Gamma-M_{1}-K-\Gamma-M_{2}-K\right)$, the upper $2 p_{y} \pi^{*}$ band and the graphite $\pi^{*}$ band intersect $E_{F}$, and form metallic states. This $2 p_{y} \pi^{*}$ band makes the hole FS around the $\Gamma$ point, while the $\pi^{*}$ band gives the electron FS around the $K$ point. These FS's are cylindrical, because the energy dispersions for these bands are very small along the $c$-axis direction $(\Gamma-A)$. In Fig. 3(b), the cross sections of FS's perpendicular to the $c$ axis are illustrated, which are simplified because their detailed shapes are not important at present. The values of these cross sections are presented in Table II in terms of the de Haas-van Alphen (dHvA) frequency. In Table III, the electron and hole carrier densities obtained numerically are also given. For model A, the carrier density of electrons is larger than that of holes. (a) $\quad \mathrm{C}_{4} \mathrm{KO}_{2} \quad$ stage-1 model A

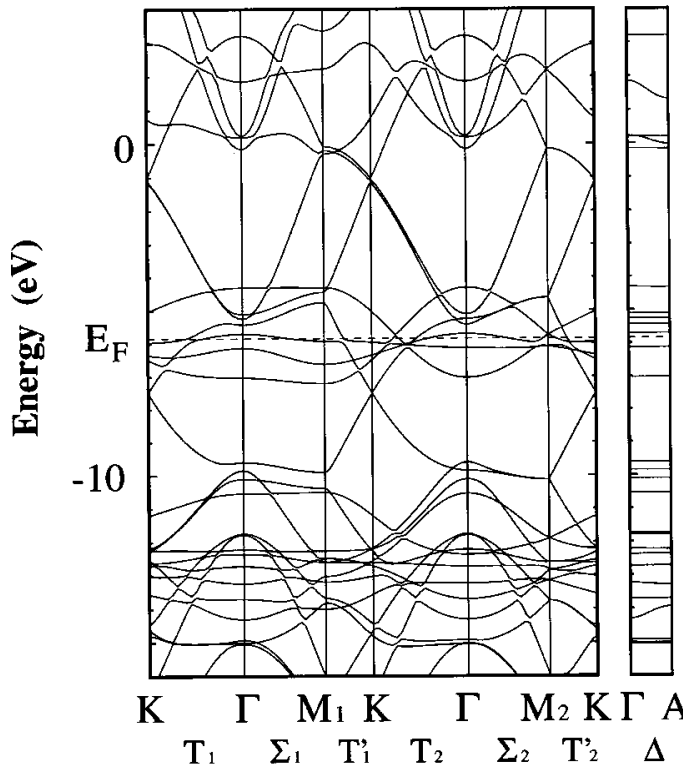

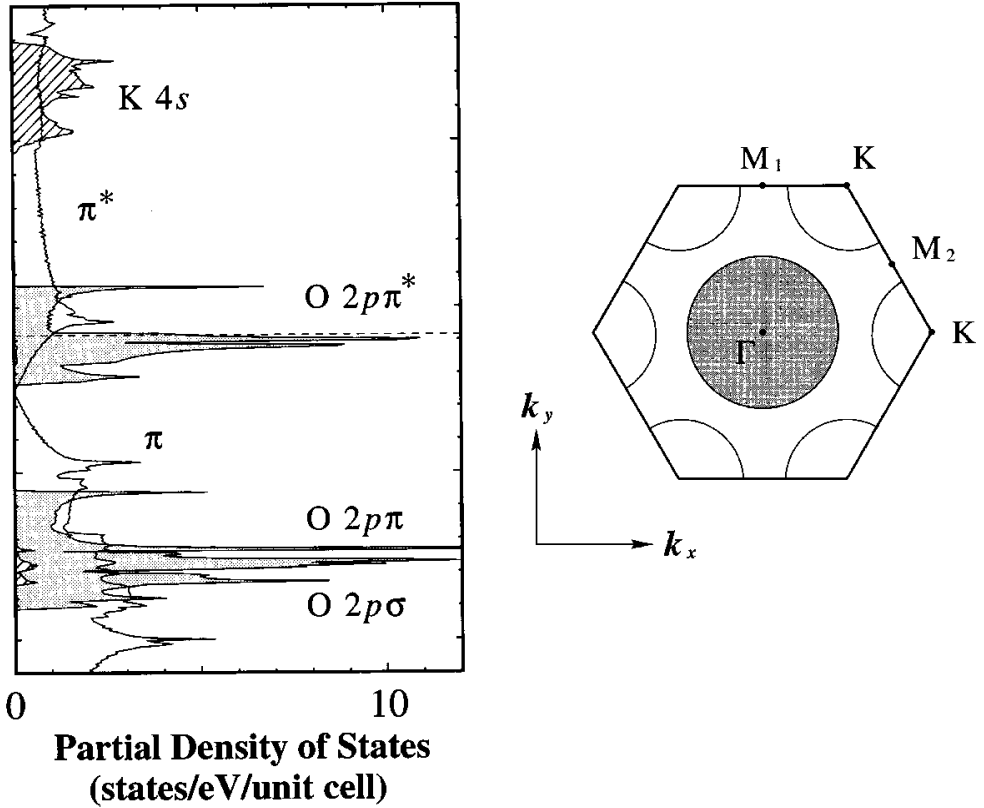

FIG. 3. (a) Band structure and corresponding partial DOS of the stage- $1 \mathrm{C}_{4} \mathrm{KO}_{2}$ with structural model A. The Fermi level is indicated by the horizontal dashed line. The partial DOS's of potassium, oxygen, and carbon are indicated by the hatched, shaded, and unshaded parts, respectively. (b) The simplified cross section of the FS perpendicular to the $c$-axis. FS's originating from the graphite $\pi^{*}$ band and oxygen $2 p$ band are represented by the unshaded and shaded parts, respectively. 
TABLE II. The cross section of the Fermi surface in terms of the dHvA frequency.

\begin{tabular}{|c|c|c|c|c|}
\hline & model A & $\begin{array}{c}\mathrm{C}_{4} \mathrm{KO}_{2} \\
\text { stage } 1 \\
\text { model } \mathrm{B}\end{array}$ & model C & $\begin{array}{c}\mathrm{C}_{8} \mathrm{KO}_{2} \\
\text { stage } 2 \\
\text { model A }\end{array}$ \\
\hline Electron (graphite) $(T)$ & 3440 & 3080 & 1750 & 912,2430 \\
\hline Hole (oxygen) $(T)$ & 4970 & & & 5070 \\
\hline
\end{tabular}

The amount of charge transfer calculated self-consistently is shown in Table IV. The $4 s$ charge of potassium is completely transferred to oxygen and carbon, i.e., potassium behaves as a donor of electron, and oxygen and carbon as acceptors. It is also found that the carbon 1 accepts larger amount of charges than the carbon 2 . This charge imbalance in the graphite layer causes the splittings of the $\pi$ and $\pi^{*}$ bands, as pointed out above. From the amount of charge transfer to oxygen atoms, the charged state of the $\mathrm{O}_{2}$ molecule is found to be $\mathrm{O}_{2}{ }^{-0.60}$.

\section{B. Stage-1 compound with structural model B}

Second, the band structure and partial DOS calculated for $\mathrm{C}_{4} \mathrm{KO}_{2}$ with structural model $\mathrm{B}$ are given in Fig. 4(a). In this model, oxygen atoms are intercalated as $\mathrm{O}_{2}$ molecules whose bond axes are perpendicular to the $c$ axis. Our results show that the $\mathrm{O}_{2}$ molecules have no magnetic moment in the compound within the accuracy of the calculation, as in model A.

The overall band structure in Fig. 4(a) is similar to that for model A. However, we should notice the different feature of the oxygen $2 p \pi^{*}$ bands near $E_{F}$. These four bands are identified as the $2 p_{z} \pi^{*}, 2 p_{y} \pi^{*}, 2 p_{z} \pi^{*}$, and $2 p_{y} \pi^{*}$ band from the higher-energy side at the $\Gamma$ point, respectively. The bandwidths of these bands are smaller than those in model A. This is due to the fact that the distance between the nearest $\mathrm{O}_{2}$ molecules is larger in model $\mathrm{B}$. This leads to a narrower and larger partial DOS near $E_{F}$, but the value of the total DOS at $E_{F}$ is smaller than that for model A, as given in Table I. This indicates that the DOS's are sensitive to the arrangement of oxygen atoms in the compound.

In the intralayer directions, the upper $2 p_{y} \pi^{*}$ band of oxygen intersects $E_{F}$, and forms the hole FS whose shape is

TABLE III. The electron and hole carrier density.

\begin{tabular}{|c|c|c|c|c|}
\hline$\langle$ theory $\rangle$ & model A & $\begin{array}{c}\mathrm{C}_{4} \mathrm{KO}_{2} \\
\text { stage } 1 \\
\text { model } \mathrm{B}\end{array}$ & model C & $\begin{array}{c}\mathrm{C}_{8} \mathrm{KO}_{2} \\
\text { stage } 2 \\
\text { model } \mathrm{A}\end{array}$ \\
\hline $\begin{array}{l}\text { Electron (graphite) } \\
\qquad\left(\times 10^{21} \mathrm{~cm}^{-3}\right)\end{array}$ & 4.05 & 3.62 & 2.07 & $0.75,2.02$ \\
\hline $\begin{array}{l}\text { Hole (oxygen) } \\
\qquad\left(\times 10^{21} \mathrm{~cm}^{-3}\right)\end{array}$ & 2.93 & 3.73 & 4.48 & 2.11 \\
\hline $\begin{array}{l}\text { Electron (oxygen) } \\
\quad\left(\times 10^{21} \mathrm{~cm}^{-3}\right)\end{array}$ & & & 2.76 & \\
\hline \multicolumn{5}{|l|}{$\langle$ experiment $\rangle$} \\
\hline $\begin{array}{l}\text { Electron (graphite) } \\
\quad\left(\times 10^{21} \mathrm{~cm}^{-3}\right)\end{array}$ & & 3.6 & & 2.6 \\
\hline
\end{tabular}

TABLE IV. The amount of charge transfer, the charge transfer averaged per one carbon atom $f_{\mathrm{C}}$, and the experimentally obtained $f_{\mathrm{C}}$.

\begin{tabular}{lcccr}
\hline \hline & & $\mathrm{C}_{4} \mathrm{KO}_{2}$ & & $\mathrm{C}_{8} \mathrm{KO}_{2}$ \\
stage 1 & & & $\begin{array}{r}\text { stage 2 } \\
\text { model A }\end{array}$ \\
& model A & model B & model C & +0.11 \\
Carbon 1 & +0.13 & +0.12 & +0.09 & 0.00 \\
Carbon 2 & +0.07 & +0.07 & +0.05 & +0.06 \\
Carbon 3 & & & & -1.00 \\
Potassium & -1.00 & -1.00 & -1.00 & +0.30 \\
Oxygen & +0.30 & +0.31 & +0.36 & +0.04 \\
$\langle$ theory & & & & \\
$f_{\mathrm{C}}$ & +0.10 & +0.10 & +0.07 & +0.04 \\
$\langle$ experiment & & & & \\
$f_{\mathrm{C}}$ & & +0.08 & & \\
\hline \hline
\end{tabular}

much different from that in model A. This hole FS is open in the $k_{y}$ direction. The cross section of this one-dimensional FS is illustrated in Fig. 4(b) together with the electron FS's originating from the $\pi^{*}$ band of graphite around the $\mathrm{K}$ point, and the corresponding $\mathrm{dHvA}$ frequency of the $\mathrm{FS}$ is given in Table II.

The amount of charge transfer is shown in Table IV. These values are nearly the same as for model A, and the charged state of the $\mathrm{O}_{2}$ molecule is $\mathrm{O}_{2}{ }^{-0.62}$. However, the carrier density of holes is slightly larger than that of electrons contrary to model A, as given in Table III.

\section{Stage-1 compound with structural model $\mathrm{C}$}

Third, we show the band structure and partial DOS calculated for $\mathrm{C}_{4} \mathrm{KO}_{2}$ with structural model $\mathrm{C}$ in Fig. 5(a). In this model, oxygen atoms are intercalated as nearly isolated ones.

In the band structure, $122 p$ bands of oxygen gather around $E_{F}$, reflecting the large distance between the nearest oxygen atoms. Above $E_{F}$, four $2 p$ bands exist in the vicinity of the $\Gamma$ point, which are identified as the $2 p_{z}, 2 p_{z}, 2 p_{x}$, and $2 p_{x}$ bands from the higher-energy side, respectively. The $2 p_{x}$ bands have the largest bandwidths due to the onedimensional arrangement of oxygen atoms in this structural model. These two $2 p_{x}$ bands of oxygen intersect $E_{F}$ together with the $\pi^{*}$ band of graphite. The upper and lower $2 p_{x}$ bands form the electron and hole FS's, respectively. The hole FS is located at the middle of the BZ, and the electron FS's exist on both sides of the hole FS [Fig. 5(b)]. These FS's are one dimensional similar to the hole FS in model B.

For model $\mathrm{C}$, the amount of charge transfer to carbon atoms is a little smaller than those for models $\mathrm{A}$ and $\mathrm{B}$ (Table IV). Accordingly, both the cross section of the electron FS and the carrier density of electrons by graphite $\pi^{*}$ band are also a little smaller (Tables II and III). On the other hand, the amount of the charge transfer to oxygen atoms is a little larger. The value of the total DOS at $E_{F}$ is smallest in the three assumed structural models (Table I).

\section{Stage-2 compound with structural model A}

Finally, the band structure and partial DOS calculated for the stage- $2 \mathrm{C}_{8} \mathrm{KO}_{2}$ with structural model $\mathrm{A}$ are shown in Fig. 
(a) $\mathrm{C}_{4} \mathrm{KO}_{2}$ stage-1 model $\mathrm{B}$

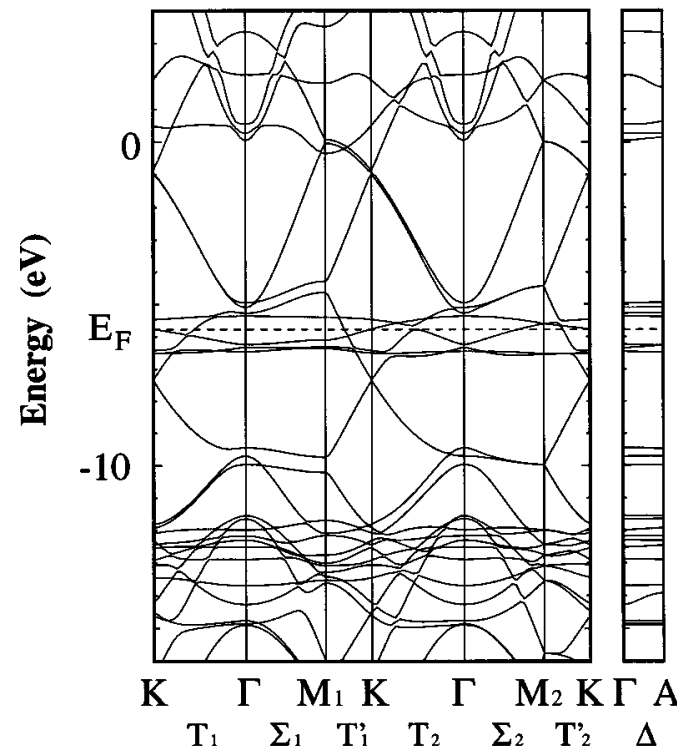

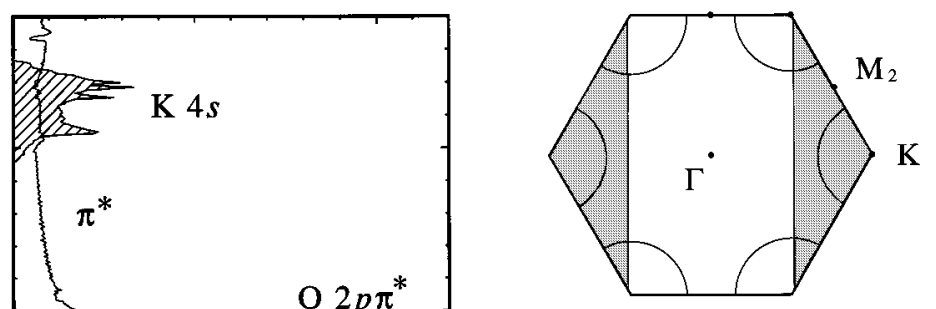

FIG. 4. (a) Band structure and partial DOS of $\mathrm{C}_{4} \mathrm{KO}_{2}$ with structural model B. (b) The cross section of the FS.

6(a). The overall band structure is similar to that for the stage- $1 \mathrm{C}_{4} \mathrm{KO}_{2}$ with model $\mathrm{A}$, except that it has two graphite bands because of the existence of two graphite layers in a unit cell. That is, the $2 p_{y} \pi^{*}$ band of oxygen intersects $E_{F}$ together with two $\pi^{*}$ bands of graphite. The $2 p_{y} \pi^{*}$ band gives the hole FS around the $\Gamma$ point, while two $\pi^{*}$ bands form two electron FS's around the $K$ point [Fig. 6(b)]. The amount of charge transfer to oxygen atoms is equal to that for the stage- 1 compound (Table IV), and the cross section of the hole FS is also nearly equal to that for the stage-1 compound (Table II). The amount of charge transfer to carbon is different for carbons 1, 2, and 3. Although carbons 1 and 3 behave as the acceptors of electron, carbon 2 is neutral. The value of the total DOS at $E_{F}$ and the carrier densities of electrons and holes are smaller than those for the stage-1 compound (Tables I and III).

\section{DISCUSSION}

For all assumed structural models, we show that the graphite $\pi^{*}$ band intersects $E_{F}$, and carbon atoms in the graphite layer accept electrons. This is the nature of the donor-type GIC. In this connection, the amount of charge transfer per one carbon atom $f_{\mathrm{C}}$ for the stage- 1 and -2 com- (a)

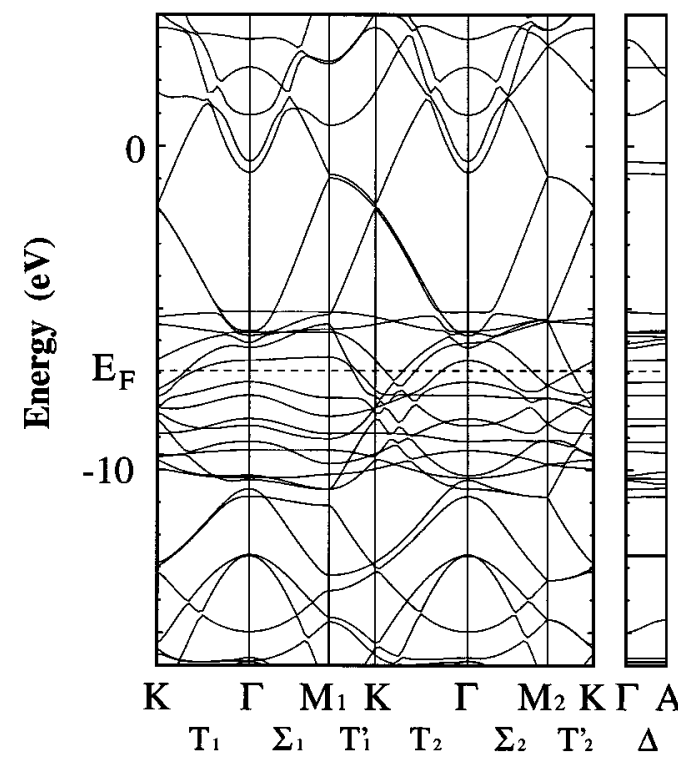

\section{$\mathrm{C}_{4} \mathrm{KO}_{2}$ stage-1 model $\mathrm{C}$}

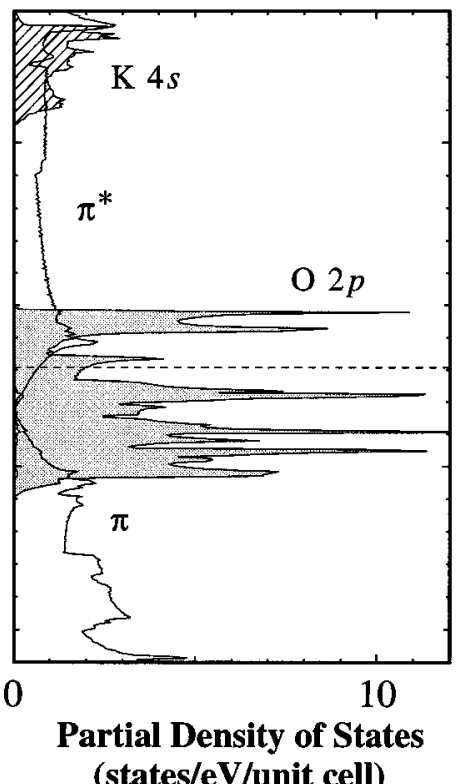

(b)

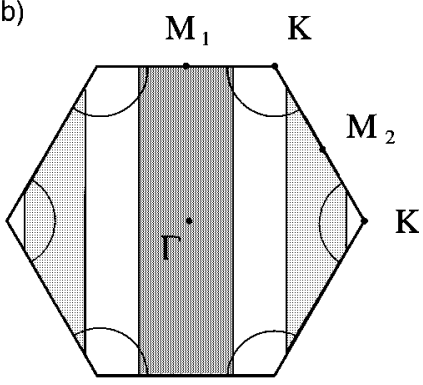

FIG. 5. (a) Band structure and partial DOS of $\mathrm{C}_{4} \mathrm{KO}_{2}$ with structural model C. (b) The cross section of the FS. The hole and electron FS's originating from the oxygen $2 p$ bands are represented by the heavy and light shaded parts, respectively. 
(a) $\quad \mathrm{CsKO}_{2} \quad$ stage-2 model $\mathrm{A}$

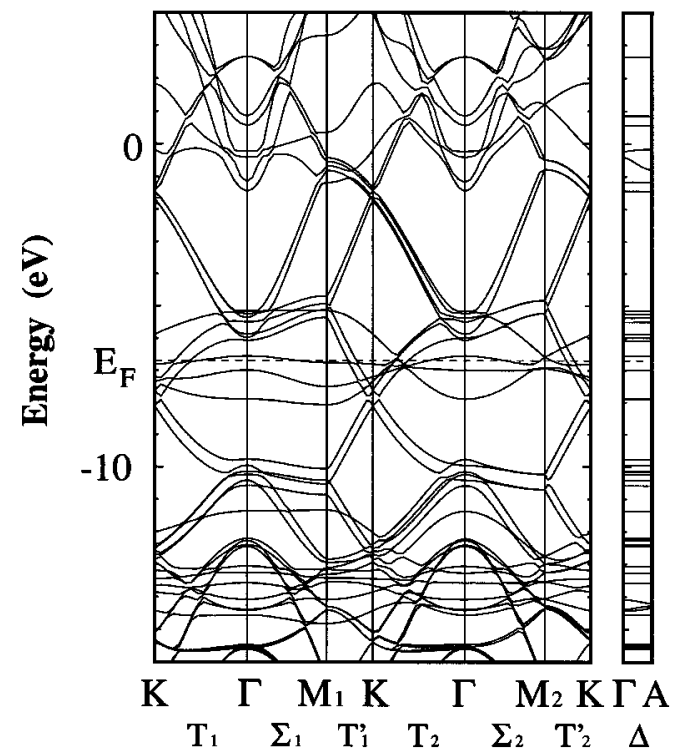

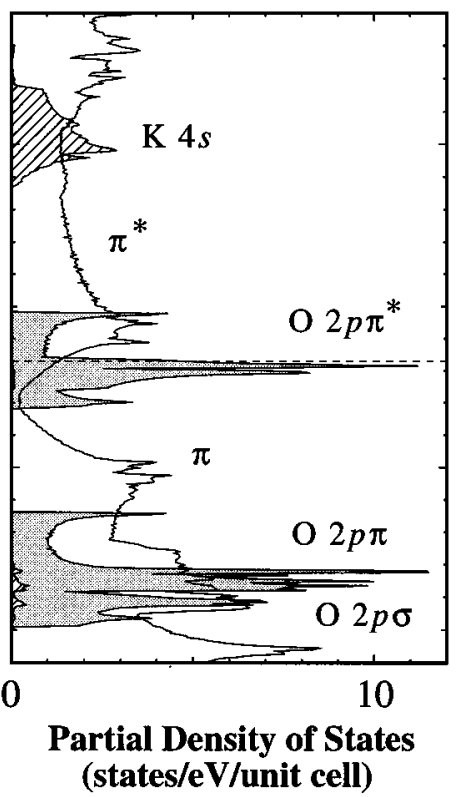

(b)

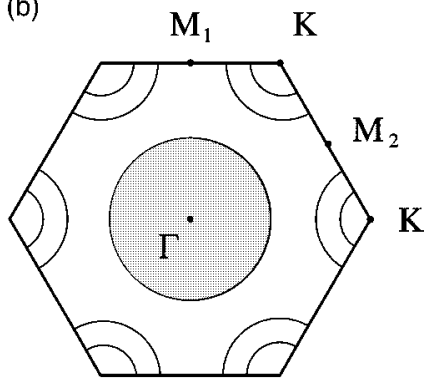

FIG. 6. (a) Band structure and partial DOS of the stage- $2 \mathrm{C}_{8} \mathrm{KO}_{2}$ with structural model A. (b) The cross section of the FS.

pounds was estimated experimentally through the $\mathrm{C}-\mathrm{C}$ interatomic distances $d_{\mathrm{C}-\mathrm{C}}$ in graphite layers by the in-plane x-ray diffraction measurements. ${ }^{4} d_{\mathrm{C}-\mathrm{C}}$ for KO-GIC's is found to be expanded compared with that of pristine graphite, as presented in Sec. II. This proves their donor-type nature, i.e., the charge transfer to carbon atoms. The values of $f_{\mathrm{C}}$ are given in Table IV for comparison with our results. The experimental $f_{\mathrm{C}}$ is in a good agreement with our theoretical $f_{\mathrm{C}}$ for each model, where the theoretical values are determined by averaging over nonequivalent carbon atoms.

From the measurements of the Pauli paramagnetic susceptibilities for the stage- 1 and -2 compounds, the values of the total DOS at $E_{F}$ were estimated, ${ }^{4}$ which are shown in Table I for comparison. These values are much larger than those expected from only the $\pi^{*}$ band of graphite, which are $0.35 \times 10^{22}$ and $0.36 \times 10^{22}$ states $/ \mathrm{eV} / \mathrm{cm}^{3}$ for the stage- 1 and -2 compounds, respectively. This fact suggests that there exist intercalate bands at $E_{F}$. This is consistent with our results, though the theoretical values differ with the structural models.

Our results show that the charge transfer to oxygen is incomplete. This leads to the coexistence of an oxygen $2 p$ band with a graphite $\pi^{*}$ band at $E_{F}$. Yamashita et al. ${ }^{5}$ measured the Hall effect for the stage-1 and -2 compounds, and found that the signs of their Hall coefficients are positive for both compounds. For donor-type GIC's, negative Hall coefficients are expected because they have electron carriers originating from the graphite $\pi^{*}$ band. Hence this experimental result suggests the existence of hole carriers originating from the intercalate bands. Moreover, the thermoelectric power measurements showed the obvious contribution of hole carriers for the stage- 1 compound. ${ }^{13}$ Yamashita et al. ${ }^{5}$ also investigated the ultraviolet photoelectron spectra of KO-GIC's, and considered from their incident photon energy dependence that the origin of the hole carriers is the oxygen $2 p$ bands. These results are also explained with our band structures. Accordingly, we believe that the electrical con- duction of KO-GIC's is undertaken by the electron carriers of the graphite $\pi^{*}$ band and the hole carriers of the oxygen $2 p$ band. In our band structures, the dispersion of the oxygen $2 p$ band is much smaller than that of the graphite $\pi^{*}$ band. Thus it is suggested that the mobility of the hole carrier is much lower than that of the electron carrier. It is also noted that the densities of both carriers are of the same order, as given in Table III. Yamashita et al. ${ }^{5}$ estimated the electron carrier densities of the graphite $\pi^{*}$ band for stage-1 and -2 compounds from the amount of charge transfer $f_{\mathrm{C}},{ }^{4}$ which is presented in Table III for comparison. Although the theoretical values differ from the structural models, the experimental and theoretical values agree on the whole. Furthermore, Yamashita et al. ${ }^{5}$ analyzed the transport properties of KOGIC's from their galvanomagnetic properties, and considered that the electrical conduction of KO-GIC's is governed by the majority electron-carriers of a graphite $\pi^{*}$ band with low mobility, and the minority hole-carriers of an oxygen $2 p$ band with high mobility. According to their estimation, the density of the hole carrier is lower by about two orders compared with that of electron carrier, while the mobility is higher by about one or two orders. Their consideration disagrees with the suggestion from our band structures. The appropriateness of their analysis, based on a simple two-band model (one electron and one hole band), should be examined for materials with complex band structures such as KOGIC's.

For all models, we see no spin polarization on oxygen atoms. This is consistent with the experimental result of the magnetic-susceptibility measurements. ${ }^{4}$ Its temperature dependence shows that there is no localized magnetic moment expected if the state of oxygen is magnetic $\mathrm{O}_{2}{ }^{-}$. Here we discuss the reason for the disappearance of the magnetic moment in KO-GIC's. In model $\mathrm{C}$, where oxygen atoms are intercalated as isolated atoms, since the charge transfer to oxygen atom is not complete, the incompletely filled oxygen $2 p$ bands form delocalized metallic states. This is why oxy- 
gen atoms have no magnetic moment. On the other hand, in models A and $\mathrm{B}$, where oxygen atoms are intercalated as $\mathrm{O}_{2}$ molecules, no magnetic moment appears for the following three reasons. The first reason is the incomplete charge transfer to the $\mathrm{O}_{2}$ molecule. Our results show that the charged state of the $\mathrm{O}_{2}$ molecule is nearly $\mathrm{O}_{2}{ }^{-0.6}$. As a consequence, the incompletely filled $2 p \pi^{*}$ band of oxygen causes the delocalized metallic states. The second reason is the dielectric screening. As is well known, for the $\mathrm{O}_{2}$ molecule, the triplet state is more stable than the singlet state due to the electronelectron interaction. The energy difference between these two states is obtained as $0.98 \mathrm{eV}$ from our spin-dependent MO calculation. However, in the compounds, this energy difference becomes much smaller because of the dielectric screening. The third reason is the crystal-field effect. The $\mathrm{O}_{2}$ molecules in the compounds interact with each other, so that the oxygen $2 p \pi^{*}$ bands near $E_{F}$ split, as seen in our band structures [Figs. 3(a) and 4(a)], where the splittings of these bands are 2.94 and $1.05 \mathrm{eV}$ at the maximum for models A and $\mathrm{B}$, respectively. These splittings of the $2 p \pi^{*}$ bands are larger than the energy difference between the triplet and singlet states of the $\mathrm{O}_{2}$ molecule, which is further reduced by the dielectric screening. This means that the crystal-field effect inhibiting the existence of magnetic moment is larger than the electron-electron interaction producing the magnetic moment. For the above reasons, oxygen atoms have no magnetic moment whether they are intercalated as isolated atoms or $\mathrm{O}_{2}$ molecules.

Our results for each structural model can explain the experimental results qualitatively. Though the value of the DOS at $E_{F}$ and the shape of the FS are quite different in each model, it is difficult to choose an adequate model in the present stage. For this purpose, a dHvA effect study is needed. Furthermore, in order to discuss more detailed electronic properties of KO-GIC's, it is necessary to clarify the detailed arrangement of oxygen atoms in the compounds.

\section{SUMMARY}

In the present paper, we constructed three structural models for the stage- 1 compound $\mathrm{C}_{4} \mathrm{KO}_{2}$ and the stage- $2 \mathrm{C}_{8} \mathrm{KO}_{2}$ based on the experimental results, and carried out selfconsistent band structure calculations. We obtained the energy bands, the DOS, the cross section of the FS, the carrier

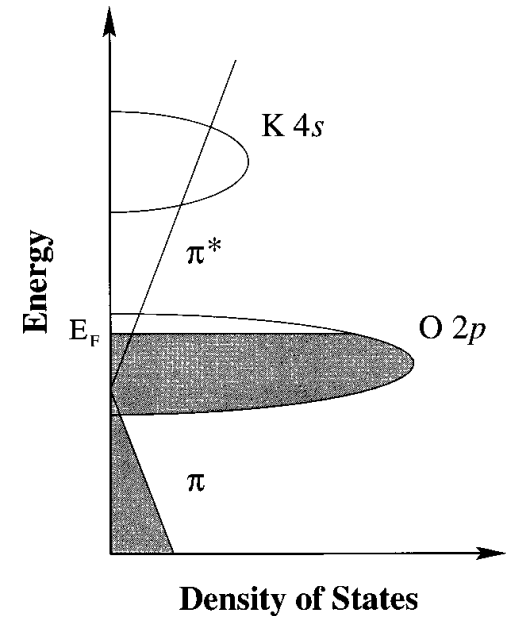

FIG. 7. The schematic of the band structure of KO-GIC's.

density, and the amount of the charge transfer among the constituent atoms. We compared these results with recent experimental results, and revealed the electronic states of KO-GIC's.

It was shown that KO-GIC's are classified into the donor type, where potassium acts as a donor and oxygen as an acceptor. The charge transfer to oxygen is incomplete. Thus the oxygen $2 p$ band coexists with the graphite $\pi^{*}$ band at $E_{F}$. The band structure of KO-GIC's can be schematically illustrated as in Fig. 7. Moreover, we showed that oxygen has no magnetic moment in these compounds within the LSDA, and discussed the reason for this.

Though the above results do not depend on the structure of the oxygen layers or the stage number, the value of the DOS at $E_{F}$ and the shape of the FS are very sensitive to the arrangement of the oxygen atoms. Thus, to discuss the electronic properties of KO-GIC's related to them, we need a detailed knowledge of the arrangement of oxygen atoms in the compounds.

\section{ACKNOWLEDGMENTS}

We would like to thank T. Yamashita and T. Enoki for providing experimental data, and for useful discussions. We are also grateful to S. Suzuki for helpful discussions.
*Present address: National Research Institute for Metals, 1-2-1 Sengen, Tsukuba 305-0047, Japan. Electronic address: higai@nrim.go.jp

${ }^{1}$ M. El Gadi, A. Herold, C. Herold, P. Lagrange, M. Lelaurain, and J. F. Mareche, Mol. Cryst. Liq. Cryst. 244, 29 (1994).

${ }^{2}$ C. Herold, M. El Gadi, J. F. Mareche, and P. Lagrange, Mol. Cryst. Liq. Cryst. 244, 41 (1994).

${ }^{3}$ V. Z. Mordkovich, Y. Ohki, S. Yoshimura, S. Hino, T. Yamashita, and T. Enoki, Synth. Met. 68, 79 (1994).

${ }^{4}$ T. Yamashita, V. Z. Mordkovich, Y. Murakami, H. Suematsu, and T. Enoki, J. Phys. Chem. Solids 57, 765 (1996).
${ }^{5}$ T. Yamashita, T. Enoki, M. Uruichi, K. Yakushi, T. Miyamae, and T. Miyazaki, J. Phys. Soc. Jpn. 66, 158 (1997).

${ }^{6}$ T. Yamashita and T. Enoki (private communication).

${ }^{7}$ S. Mizuno, H. Hiramoto, and K. Nakao, J. Phys. Soc. Jpn. 56, 4466 (1987).

${ }^{8}$ S. Mizuno and K. Nakao, Phys. Rev. B 41, 4938 (1990).

${ }^{9}$ P. Hohenberg and W. Kohn, Phys. Rev. 136, B864 (1964).

${ }^{10}$ W. Kohn and L. J. Sham, Phys. Rev. 140, A1133 (1965).

${ }^{11}$ J. P. Perdew and A. Zunger, Phys. Rev. B 23, 5048 (1981).

${ }^{12}$ A. Zunger and A. J. Freeman, Phys. Rev. B 15, 4716 (1977).

${ }^{13}$ T. Yamashita, T. Enoki, T. Miyamae, and T. Miyazaki, Mol. Cryst. Liq. Cryst. (to be published, 1998). 